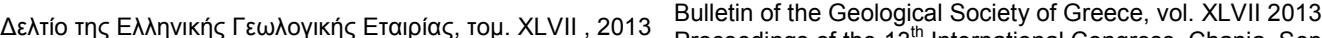

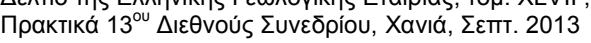

\title{
A MARKOV MODEL FOR SEISMIC HAZARD ANALYSIS ALONG THE HELLENIC SUBDUCTION ZONE (GREECE)
}

\author{
Votsi I. ${ }^{1,2}$, Tsaklidis G. ${ }^{2}$, Limnios N. ${ }^{1}$, Papadimitriou E. ${ }^{3}$ and Vallianatos F. ${ }^{4}$ \\ ${ }^{1}$ Université de Technologie de Compiègne, Laboratoire de Mathématiques Appliquées de \\ Compiègne,irene.votsi@utc.fr,nikolaos.limnios@utc.fr \\ 2 Aristotle University of Thessaloniki,Department of Mathematics, tsaklidi@math.auth.gr \\ ${ }^{3}$ Aristotle University of Thessaloniki, Geophysics Department, ritsa@geo.auth.gr \\ ${ }^{4}$ Technological Educational Institute of Crete, Laboratory of Geophysics and Seismology, \\ fvallian@chania.teicrete.gr
}

\begin{abstract}
A homogeneous finite-state discrete-time Markov model is applied for the earthquake occurrence in the Hellenic Subduction Zone (Greece), a region accommodating high seismic activity, being a key structure from a seismotectonic point of view. An attempt is made to provide a stochastic representation of the earthquake process and to assess the seismic hazard through the application of the Markov model. The model is applied on a complete data sample comprising strong ( $M \geq 5.5)$ earthquakes that occurred in the study area since 1911 up to present. The continuous magnitude scale is divided into appropriate intervals to specify discrete states of the model. As the stochastic behavior of the model is governed by its transition probability matrix, we firstly estimate its well-known maximum likelihood estimator. The estimation of the transition probability matrix leads to the estimation of important indicators of the Markov chain, including hitting times and failure rate functions. The mean number of steps for the first occurrence of an anticipated earthquake (belonging to the class with the stronger events, which we are more interested in) is estimated along with its variance. In a next step, we calculate the confidence interval of the aforementioned estimators.
\end{abstract}

Key words: transition probabilities, earthquake occurrence probabilities, confidence intervals.

\section{Пєрі́ $\eta \psi \eta$}

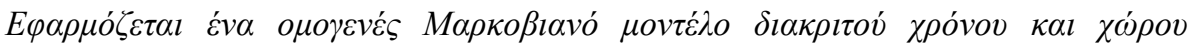

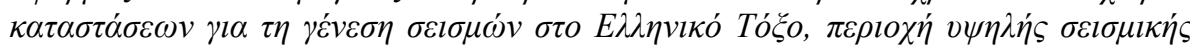

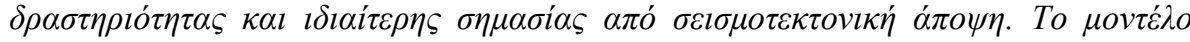

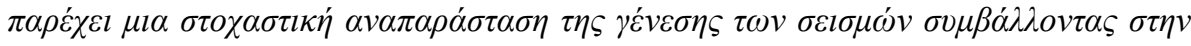

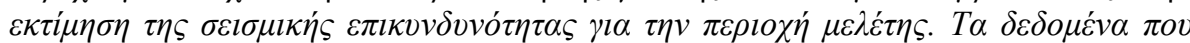

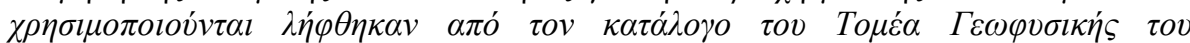

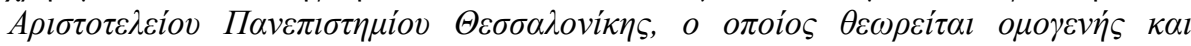

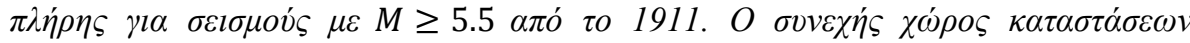

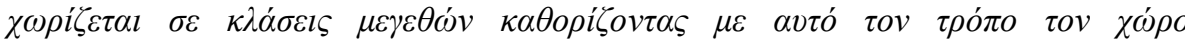

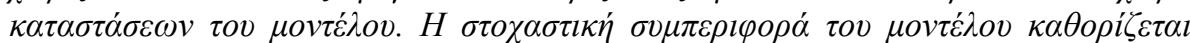

$\underline{\text { XLVII. No } 3-1376}$ 


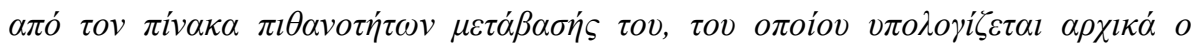

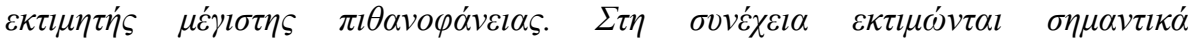

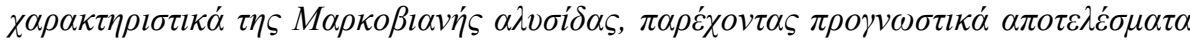

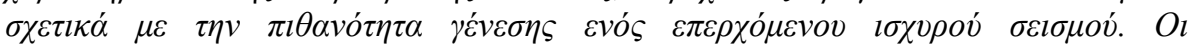

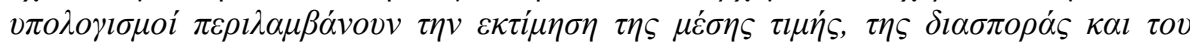

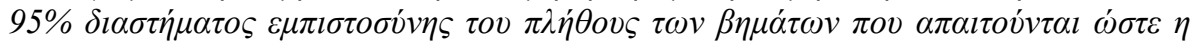

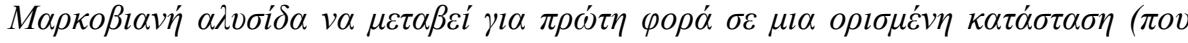

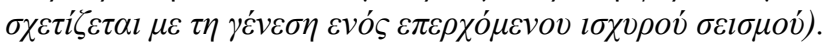

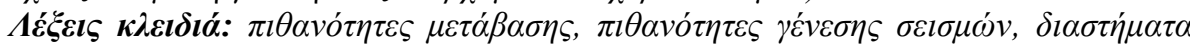
$\varepsilon \mu \pi \imath \sigma \tau о \sigma \dot{v} \eta \varsigma$.

\section{Introduction}

Until now deterministic approaches are not able to describe the earthquake dynamics appropriately. One reason for this is the limited access to important state variables of the underlying processes (e.g., stresses and material properties). Comparing the deterministic approach with the probabilistic one, we should note that the latter is the one most in favor today, relying on stochastic models to compute occurrence probabilities of strong earthquakes. Stochastic earthquake occurrence models are divided to memoryless and with-memory ones, where memory refers to time, size or location of preceding events. Usual probabilistic seismic hazard assessment is based on the assumption that the earthquake process is memoryless. This assumption is made by considering that the times of events above some threshold magnitude consist a realization of a time-homogeneous Poisson process. The Poisson model provides earthquake occurrence probabilities in a given period independently of the elapsed time since the previous one. The hazard rate describing the instantaneous earthquake occurrence rate at any time is constant confirming the stationary nature of the Poisson assumption. This is not in accordance with elastic rebound principles (Reid, 1910) and characteristic earthquake theory, which imply that if the accumulated strain of a fault section is released in an earthquake, then the initial value of the hazard should be zero and started to increase up to the next event.

Markov models were engaged for seismic hazard assessment since 1980. They are stochastic models with memory, where memory refers to the magnitude of the preceding event (one-step memory case). This type of memory can be incorporated into the definition of their state space via earthquake magnitudes. According to previous studies, it is observed that whereas the Poisson model may be applied to regions characterized by moderate frequent earthquakes, Markov models describe the sequences of events more adequately at regions with strong infrequent events (Anagnos and Kiremidjian, 1988 and the references therein), a very important implication for continental regions where there is shortage of multiple recurrent events into the same fault segment. The latter authors reviewed the basic assumptions of the various models, summarized their stochastic representations and discussed the parameters necessary for applications. Tsapanos and Papadopoulou (1999) applied a discrete-time Markov model for earthquake occurrences, in one of the most seismically active regions of the world, the area of Alaska and Aleutian Islands. The states of the model were defined on the basis of seismic zones (Papadimitriou, 1994). The frequency of visits and the transition probabilities in each one of the defined states were calculated for different magnitude thresholds. In a next step, the model was considered to visit an "active" or an "inactive" state. For each seismic zone, the transition probabilities between the active and inactive states were calculated along with the mean duration of an active period.

Here we focus on the application of a discrete-time Markov model, which assumes that the state duration is geometrically distributed depending on the current state of the Markov chain. Important indicators of the model are estimated, aiming to provide earthquake forecasting results. The transition probabilities of the chain are estimated along with its relevant measures, resulting to the calculation of earthquake occurrence probabilities. The Hellenic Arc is selected for this investigation because it exhibits high seismic activity, and therefore hazard assessment in this area

$\underline{\text { XLVII, No } 3-1377}$ 
is of paramount importance. An adequate number of strong $(M \geq 6.4)$ earthquakes, along with an adequate number of moderate $(M \geq 5.5)$ events is available since 1911 (Figure 1). It is the first time that stochastic models are applied in the study area, and in particular the Markov model.

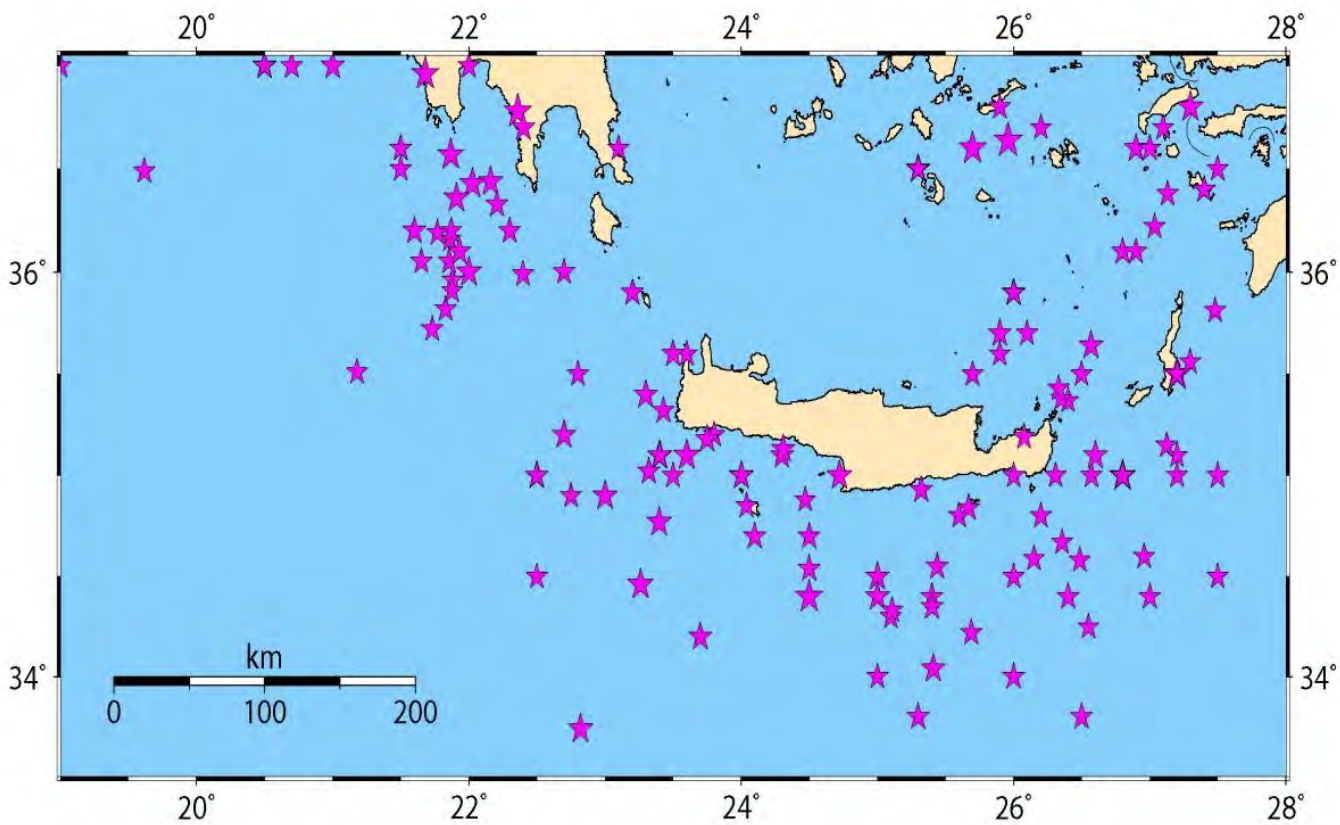

Figure 1 - Epicentral distribution of the earthquakes with $M \geq 5.5$ that occurred in the study area during $1911-2012$.

\section{Seismotectonic Setting}

Eastern Mediterranean active tectonics is dominated by slow convergence of Africa and Europe of about $1 \mathrm{~cm} / \mathrm{yr}$ (Argus et al., 1989) that started about $83 \mathrm{Ma}$ ago (Olivet et al., 1982) and lateral westward escapement of Anatolian microplate (McKenzie, 1978). In the eastern Mediterranean, the southern Aegean constitutes an area of intense seismic activity and in particular along the Hellenic Arc (Figure 2), which has been recognized as a subduction zone with a $30^{\circ}-45^{\circ}$-dipping Wadati-Benioff seismic plane (Papazachos and Comninakis, 1971). The evolution of the orogen back-arc basin system of the Hellenic-Aegean system is qualitatively explained by slab detachment at crust-lithosphere levels, and specifically lateral migration of the tear in the slab along the strike of the subduction zone. This provides a feasible mechanism for slab roll-back and arc migration, for orogenic collapse (and concurrent creation of large sedimentary depo-centers), and for changing chemical signatures of arc volcanism due to replacement of magma source regions by asthenospheric material (Wortel and Spakman, 1992).

Extension behind the subduction system began in the Middle to Late Miocene (10 - $13 \mathrm{Ma})$ (LePichon and Angelier, 1979; Jackson, 1994) or possibly as recently as $6 \mathrm{Ma}$ (McKenzie, 1978). Extension of up to 100 per cent is thought to have affected the Aegean region in a north-south direction (McKenzie, 1978), with the greatest extensional strains located in the southern Aegean, north of Crete (Angelier et al., 1982; Jackson, 1994). Between the Hellenic trench and Africa, most of the region is comprised by the Mediterranean Ridge (MR), which terminates near the Calabrian arc in the west and south of Anatolia in the east. The Hellenic arc is laterally bounded by Subduction-Transform Edge Propagators (STEP), kinks in the plate boundary that are ongoing tearing of oceanic lithosphere near the horizontal terminations of subduction trenches (Govers and Wortel, 2005), the dextral Cephalonia Transform Fault in the west (Scordilis et al., 1985) and the 
sinistral Rhodos fault in the east (Papazachos and Papazachou, 2003). Its western part is rectilinear and exactly perpendicular to the direction of underthrusting of the sea floor accommodating the largest known earthquake $(M=8.3)$ that has occurred in the Mediterranean region (Papazachos and Papazachou, 2003; Papadimitriou and Karakostas, 2008), while the eastern one is more complex and approximately parallel to it (Figure 2). The stresses related to subduction maintain the rectilinear boundary along the western convergent zone, while this containment does not exist on the eastern, mostly transform boundary (LePichon and Angelier, 1979).

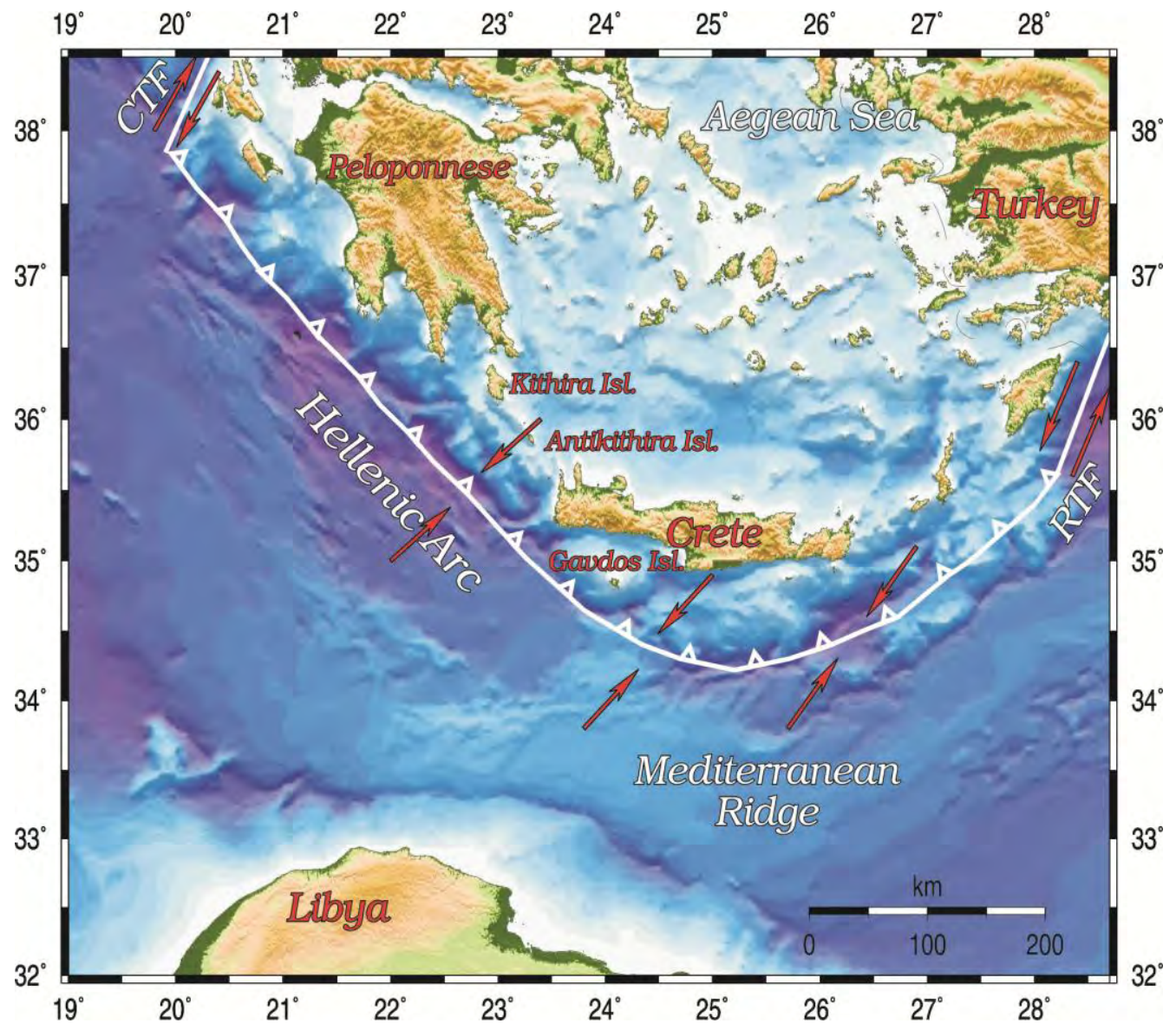

Figure 2 - Morphology and main seismotectonic properties of the study area.

\section{The Model}

A Markov chain describes a stochastic process where transitions between states are governed by probability distributions. More formally, a Markov chain is a sequence of random variables $\boldsymbol{J}:=\left(J_{n}\right)_{n \in N}$, where if the value $J_{n}=i$ is known, then the future evolution of the chain depends only on the last visited state $i$ and it is stochastically independent of the visited states $J_{n-1}, \ldots, J_{0}$ ("Markov property"). We denote by $E$ the state space of the Markov chain, which is considered to be a finite one. The initial probability distribution of the chain is denoted by $\boldsymbol{a}:=(a(i) ; i \in E)$ (row vector), where $a(i):=P\left(J_{0}=i\right)$, whereas its transition probability matrix is denoted by $\boldsymbol{P}:=(p(i, j) ; i, j \in E)$, where $p(i, j):=P\left(J_{n+1}=j \mid J_{n}=i\right)$, for all $n \in N$. The transition probabilities are assumed to be independent of $n$, that is the chain is time-homogeneous. 
For fixed time M, we define by

\section{Equation 1 - Formula for the First Counting Process}

$N_{\mathrm{M}}(i, j):=\sum_{n=1}^{\mathrm{M}} 1_{\left\{J_{n-1}=i, J_{n}=j\right\}}$,

the number of transitions from state $i$ to state $j$ up to time $\mathrm{M}$, whereas the number of visits to state $i$ up to time $\mathrm{M}$ is defined by:

\section{Equation 2 - Formula for the Second Counting Process}

$N_{\mathrm{M}}(i):=\sum_{n=1}^{\mathrm{M}} 1_{\left\{J_{n}=i\right\}}$.

The maximum likelihood function at time $\mathrm{M}$ is expressed in terms of the transition probabilities as follows:

\section{Equation 3 - Formula for the Likelihood Function}

$L_{\mathrm{M}}(\boldsymbol{P})=a\left(J_{0}\right) p\left(J_{0}, J_{1}\right) \cdots p\left(J_{\mathrm{M}-1}, J_{\mathrm{M}}\right)=a\left(J_{0}\right) \prod_{(i, j) \in E^{2}}(p(i, j))^{N_{\mathrm{M}}(i, j)}$.

By maximizing the corresponding log-likelihood function, we obtain the maximum likelihood estimator (MLE) of the transition probability $p(i, j)$ written as follows (see, e.g., Billingsley, 1961):

\section{Equation 4 - Formula for the MLEs of the Transition Probabilities}

$\hat{p}_{\mathrm{M}}(i, j)=\frac{N_{\mathrm{M}}(i, j)}{N_{\mathrm{M}}(i)}$,

for all $i, j \in E$.

\section{Results}

Concerning states classification, according to previous studies, the states of the Markov chain can be considered to be either the magnitudes or energy release levels of earthquakes. In our study the continuous magnitude scale is divided into appropriate intervals to specify discrete states visited by the Markov chain. In particular, we use three states corresponding to data magnitude intervals: State 1: [5.5, 5.6], State 2: [5.7, 6.0], State 3: [6.1, 7.5].

In the sequel, the discrete-time Markov model is applied to the above mentioned earthquake catalogue and the number of observed transitions in the dataset from each state $i$ to each state $j$ $(i, j \in E)$ are presented as elements of the following matrix:

$$
\text { Trans }=\left(\begin{array}{ccc}
38 & 23 & 12 \\
19 & 12 & 11 \\
16 & 6 & 7
\end{array}\right)
$$

Considering the earthquake sequence as a trajectory of the Markov chain in $[0, \mathrm{M}] \subset N$ we estimate the transition probability matrix $\hat{P}_{\mathrm{M}}=\left(\hat{p}_{\mathrm{M}}(i, j)\right)$ (Table 1$)$, which governs the stochastic behavior of the Markov chain. The elements of the transition probability matrix describe the probabilities of the occurrence of an earthquake with a certain magnitude state, given that an earthquake with a certain magnitude state occurred. We go one step further and calculate the 95\% confidence intervals of the transition probabilities resulting from the parametric bootstrap method. More specifically, the bootstrap confidence intervals are computed by means of the percentile method (Efron and Tibshirani, 1993) and they allow us to estimate the range of the values under consideration. The lower confidence bound (L.C.B.) is the $b \cdot a / 2$-th order value of the transition probability, the upper confidence bound (U.C.B.) is the $b \cdot(1-a) / 2$-th order value, where $b$ denotes the number of bootstrap samples and $a / 2,(1-a) / 2$, stand for the percentiles of the 
estimated quantity. New data sets (1000 in size) with each one containing 145 data points (the same size as the original dataset) are generated by using the MLEs of the transition probabilities. Then the model is applied to each dataset resulting in an empirical distribution around the MLEs of the transition probabilities and leading to the calculation of their $95 \%$ confidence intervals (Table 1).

Table 1 - Bootstrap percentile confidence intervals at $95 \%$ level of confidence for the transition probabilities of the Markov model.

\begin{tabular}{|c|l|l|l|l|l|l|l|l|l|}
\hline & $\hat{p}_{\mathrm{M}}(1,1)$ & $\hat{p}_{\mathrm{M}}(1,2)$ & $\hat{p}_{\mathrm{M}}(1,3)$ & $\hat{p}_{\mathrm{M}}(2,1)$ & $\hat{p}_{\mathrm{M}}(2,2)$ & $\hat{p}_{\mathrm{M}}(2,3)$ & $\hat{p}_{\mathrm{M}}(3,1)$ & $\hat{p}_{\mathrm{M}}(3,2)$ & $\hat{p}_{\mathrm{M}}(3,3)$ \\
\hline MLE & 0.5205 & 0.3151 & 0.1644 & 0.4524 & 0.2857 & 0.2619 & 0.5517 & 0.2069 & 0.2414 \\
\hline L.C.B. & 0.3881 & 0.2169 & 0.0870 & 0.3111 & 0.1351 & 0.1400 & 0.3846 & 0.0741 & 0.0769 \\
\hline U.C.B. & 0.6267 & 0.4286 & 0.2540 & 0.6000 & 0.4107 & 0.4054 & 0.7308 & 03600 & 0.3846 \\
\hline
\end{tabular}

In what follows earthquake occurrence probabilities are calculated following Sadek and Limnios (2002). Let us denote by $1_{s, r}=(1, \ldots, 1,0, \ldots, 0)^{T}$ the $s$-dimensional column vector whose $r$ first elements are equal to 1 and the others are equal to 0 . We further denote $1_{r}=1_{r, r}$. At a next step we calculate the probability of the occurrence of an earthquake with $M \in[5.5,6.0]$ at time $n \in N$, independently of the fact that an earthquake has occurred or not in $[0, n)$, which is defined as

\section{Equation 5 - Formula for the Probability}

$A(n)=P\left(J_{n} \in\{1,2\}\right)=a P^{n} 1_{s, r}, n \in N$,

with corresponding maximum likelihood estimator:

\section{Equation 6 - Formula for the MLE of the Probability}

$\hat{A}_{\mathrm{M}}(n)=\hat{a} \hat{P}_{\mathrm{M}}^{n} 1_{s, r}$,

where $\hat{P}_{\mathrm{M}}^{n}=\left(\hat{P}_{\mathrm{M}}\right)^{n}, n \in N$, and $1_{s, r}=1_{3,2}$. Figure 3 represents the quantity under study along with its $95 \%$ confidence interval. This figure leads us to the conclusion that the occurrence probability of an earthquake with $M \in[5.5,6.0]$ takes the constant value 0.792 after four steps $(n=4)$, given that the previous occurrences are not taken into account. The same conclusion is reached concerning its $95 \%$ confidence interval.

We further denote by $P_{11}$ the restriction of the transition probability matrix to the set of states $\{1,2\}$ and by $\hat{P}_{\mathrm{M} 11}$ its MLE. The probability of the occurrence of an earthquake with $M \in[5.5,6.0]$ at time $n$ and that up to time $n$ there was no earthquake occurrence with $M \leq 6.0$, is given by

\section{Equation 7 - Formula for the Probability}

$R(n)=P\left(J_{u} \in\{1,2\}, \forall u \in[0, n]\right)=a_{1} P_{11}^{n} 1_{r}, n \in N$,

with corresponding maximum likelihood estimator:

\section{Equation 8 - Formula for the MLE of the Probability}

$\hat{R}_{\mathrm{M}}(n)=\hat{a}_{1} \hat{P}_{\mathrm{M} 11}^{n} 1_{r}$.

Figure 4 represents the MLE of $R(n)$, along with its $95 \%$ confidence interval. In this figure we can see that as time elapses, the target-probability decreases. This result was expected in the sense that as time increases the occurrence probability of a stronger event $(M>6.0)$ increases, decreasing by this way the values of $\hat{R}_{\mathrm{M}}(n)$. After a certain number of steps, $\hat{R}_{\mathrm{M}}(n)$ and its confidence interval tend to zero. In other words, after a certain number of steps, the occurrence probability of an earthquake with $M>6.0$ and its confidence interval tend to one. Let us now define as hitting time the occurrence time of an earthquake with $M \geq 6.1$ ("failure") denoted by $T$. The conditional 
probability of the occurrence of an earthquake with $M \geq 6.1$ at time $n$, given that only earthquakes with $M \in[5.5,6.0]$ occurred up to time $n-1$, is given by

\section{Equation 9 - Formula for the Conditional Probability}

$\lambda(n)=P(T=n \mid T \geq n)=\left\{\begin{array}{c}1-\frac{R(n)}{R(n-1)}, \quad R(n-1) \neq 0, \\ 0, \quad \text { otherwise, }\end{array}\right.$

for every $n \in N^{*}$.

The maximum likelihood estimator for the target probability (known as BMP-failure rate), for every $n \in N^{*}$, is given by

\section{Equation 10 - Formula for the MLE of the Conditional Probability}

$\hat{\lambda}_{\mathrm{M}}(n)=\left\{\begin{array}{c}1-\frac{\hat{R}_{\mathrm{M}}(n)}{\hat{R}_{\mathrm{M}}(n-1)}, \quad \hat{R}_{\mathrm{M}}(n-1) \neq 0, \\ 0, \quad \text { otherwise. }\end{array}\right.$

and $\hat{\lambda}_{\mathrm{M}}(0)=1-\hat{R}_{\mathrm{M}}(0)$. Figure 5 depicts the MLE and the $95 \%$ confidence interval of the quantity under study. These results are significant in the sense that they take into account the history of earthquake occurrences up to time $n$ in order to provide occurrence probabilities of strong events for time $n$. In particular, the probability of the occurrence of an earthquake with $M>6.0$ at time $n$, given that only earthquakes with $M \in[5.5,6.0]$ occurred up to time $n-1$, takes the constant value 0.202 after the fourth step.

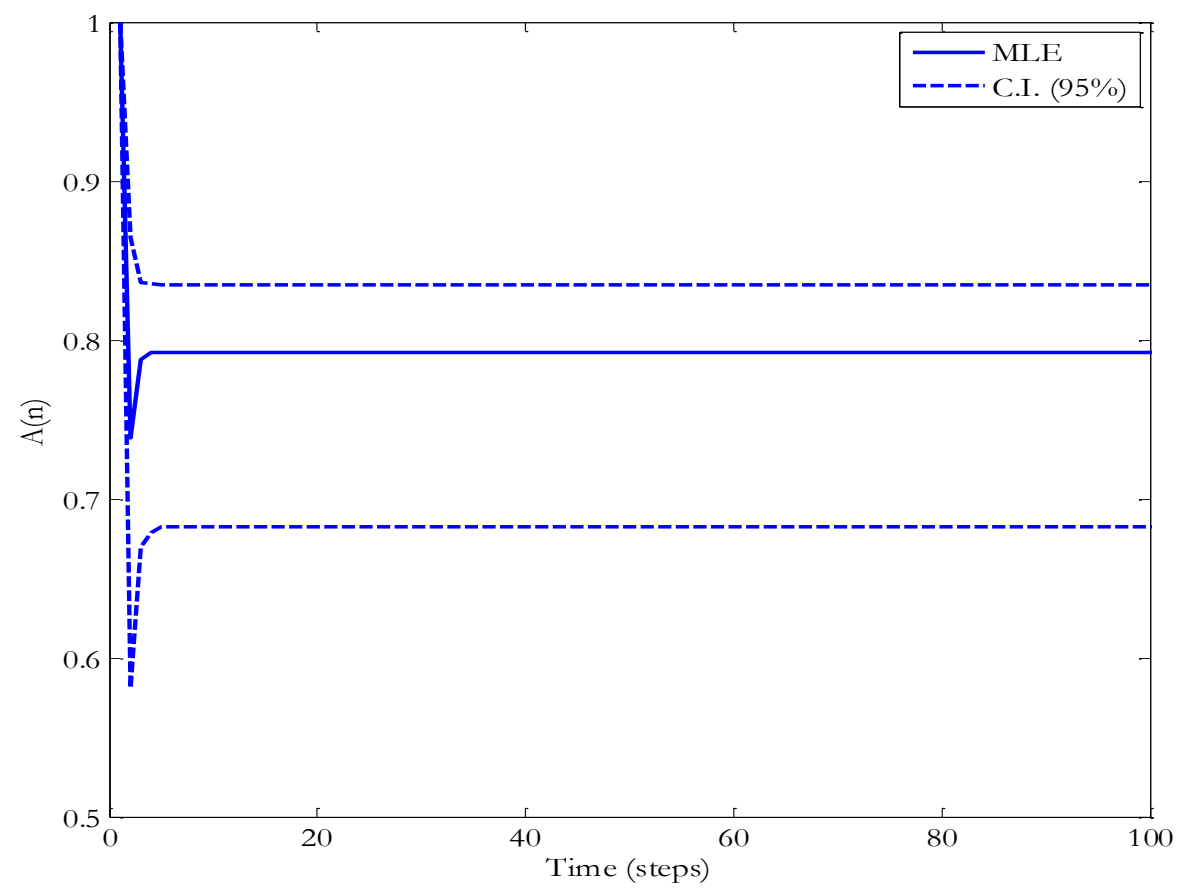

Figure 3 - Maximum likelihood estimator and $95 \%$ confidence interval for $A(n)$.

XLVII, No 3 - 1382 
Remark. At this point we should note that although we work at the discrete-time case, we joined the values continuously, in order to observe their evolution in time more clearly.

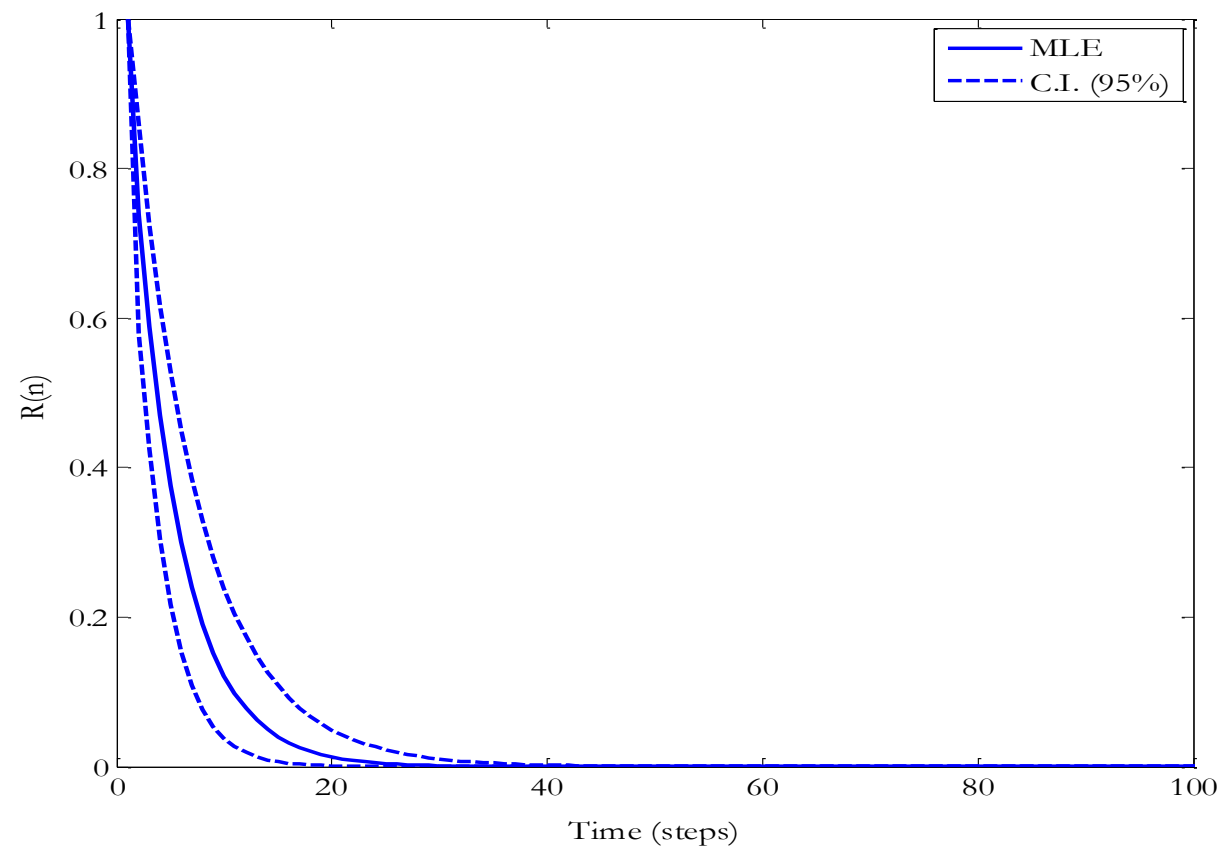

Figure 4 - Maximum likelihood estimator and 95\% confidence interval for $R(n)$.

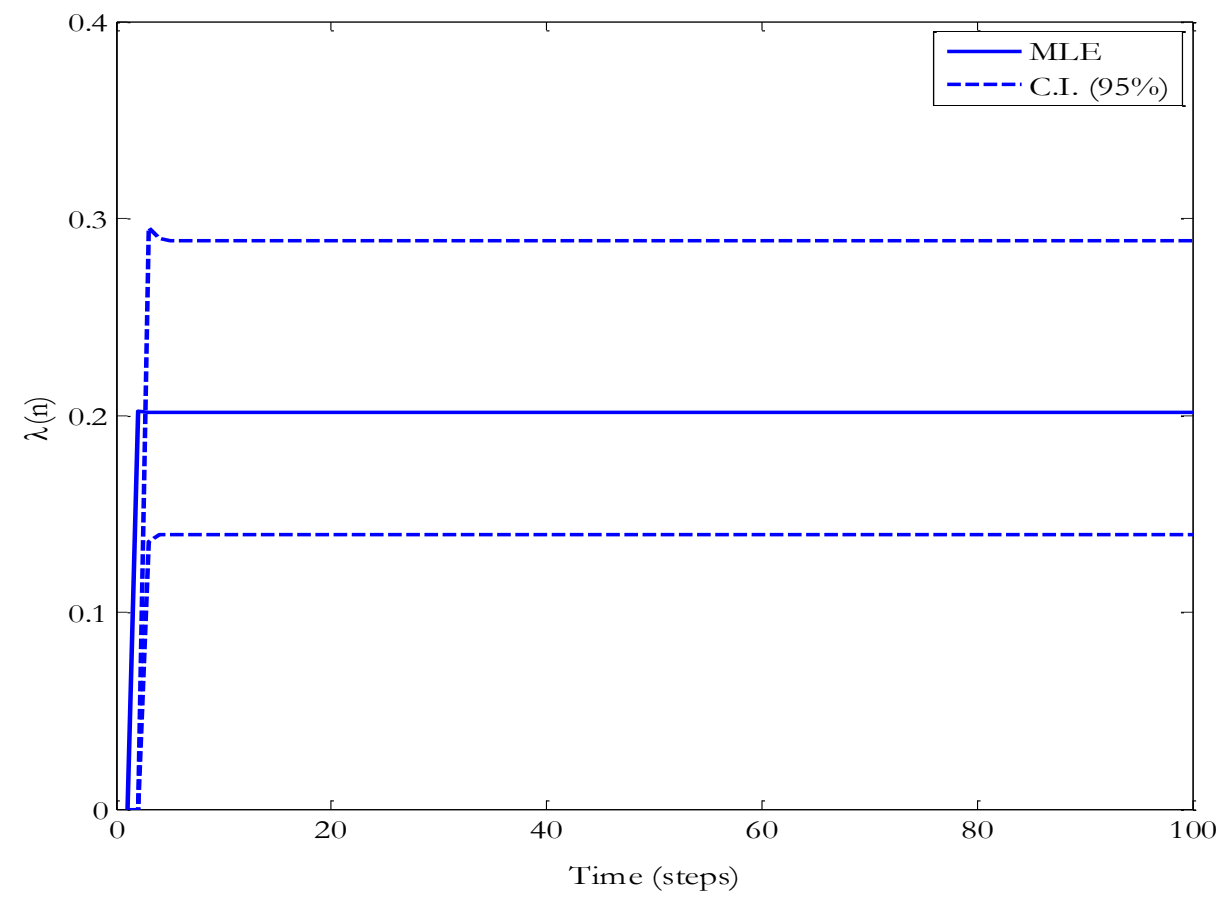

Figure 5 - Maximum likelihood estimator and $95 \%$ confidence interval for $\lambda(n)$.

$\underline{\text { XLVII, No } 3-1383}$ 
Let us consider a partition $\boldsymbol{U}, \boldsymbol{D}$ of the state space $\boldsymbol{E}$, i.e., $\boldsymbol{E}=\boldsymbol{U} \cup \boldsymbol{D}$, with $\boldsymbol{U} \cap \boldsymbol{D}=\varnothing, \boldsymbol{U} \neq \emptyset$ and $\boldsymbol{D} \neq \varnothing$. The random variable $\boldsymbol{T}_{\boldsymbol{i}}$, representing the number of steps for visiting for the first time a state $\boldsymbol{i}^{\prime} \in \boldsymbol{D}$ is defined by $\boldsymbol{T}_{\boldsymbol{i}},:=\boldsymbol{i n f}\left\{\boldsymbol{n} \in \boldsymbol{N}: \boldsymbol{J}_{\boldsymbol{n}}=\boldsymbol{i}^{\prime}\right\}$. The mean number of steps for visiting for the first time the state $\boldsymbol{i}^{\prime} \in \boldsymbol{D}$ starting from a state belonging to $\boldsymbol{U}$ is given by the formula (Kemeny and Snell, 1976) $\boldsymbol{E}\left[\boldsymbol{T}_{\boldsymbol{i}}\right]=\boldsymbol{a}_{\mathbf{1}}\left(\boldsymbol{I}-\boldsymbol{P}_{\mathbf{1 1}}\right)^{-\mathbf{1}} \mathbf{1}_{\boldsymbol{r}}$. Index 1 means restriction of the transition probability matrix on $\boldsymbol{U}$, i.e., $\boldsymbol{P}_{\mathbf{1 1}}$ means restriction of the matrix $\boldsymbol{P}$ on $\boldsymbol{U} \times \boldsymbol{U}, \boldsymbol{a}_{\mathbf{1}}$ denotes the restriction of the raw vector $\boldsymbol{a}$ in $\boldsymbol{U}, \boldsymbol{r}$ is the number of states in $\boldsymbol{U}$ and $\mathbf{1}_{\boldsymbol{r}}$ stands for the column vector of $\boldsymbol{r}$ ones. Let us now consider that set $\boldsymbol{U}$ contains all the states of $\boldsymbol{E}$ apart from the state $\boldsymbol{i}^{\prime}$, which corresponds to the occurrence of an earthquake with $\boldsymbol{M} \geq \mathbf{6 . 1}$, i.e., the occurrence of an earthquake that belongs in the third magnitude state. The maximum likelihood estimator of the target number is given by the formula

\section{Equation 11 - Formula for the MLE of the Mean Number of Steps}

$$
E \widehat{\left[T_{l^{\prime} \mathrm{M}}\right]}=\hat{a}_{1}\left(I-\hat{P}_{\mathrm{M} 11}^{n}\right)^{-1} \mathbf{1}_{r} \text {. }
$$

Given that the current state of the Markov chain is $J_{0}=2$, the mean number of steps for the first occurrence of an anticipated earthquake with $\boldsymbol{M} \geq \mathbf{6 . 1}$ is 4.661 . In other words, given that the last earthquake that occurred was of magnitude $\boldsymbol{M} \in[\mathbf{5 . 7}, \mathbf{6 . 0}]$, the first occurrence of an earthquake with $\boldsymbol{M} \geq \mathbf{6 . 1}$ is expected in an average number of 4.661 steps. The corresponding $95 \%$ bootstrap confidence interval turns out to be $(3.270,6.993)$.

\section{Conclusions}

The objective of this study is to describe a Markov model for characterizing the occurrence of strong $(M \geq 5.5)$ earthquakes along the Hellenic Arc, which is consistent with the general physical process contributing to their occurrence. Contrary to the previous studies, where Markov models were applied as earthquake occurrence models, here we go one step further and estimate earthquake occurrence probabilities along with their $95 \%$ confidence intervals. The model provides important measures of the Markov chain leading to probabilistic seismic hazard results. It could serve as a basis for comparison with other stochastic models such as renewal ones as well as with more complicated models such as semi-Markov models. More accurate forecasting results could be feasible by the inclusion of the temporal and the spatial components to the model. Examples of extension are based on the assumption that the Markov chain is non-homogeneous or is a higher-order Markov chain, allowing second- or higher-order Markov dependence. The current research could be enriched by the calculation of other measures derived from the transition probability matrix (e.g., moments of the number of steps for the first earthquake occurrence of a certain magnitude state) to provide additional results. Alternative methods could be applied for the calculation of the confidence intervals for the quantities under study, and the derived results could be compared with the initial ones.

\section{Acknowledgements}

This work was supported by the THALES Program of the Ministry of Education of Greece and European Union in the framework of the project entitled "Integrated understanding of Seismicity, using innovative Methodologies of Fracture mechanics along with Earthquake and non extensive statistical physics - Application to the geodynamic system of the Hellenic Arc. SEISMO FEAR HELLARC". Geophysics Department, AUTH, contribution number 815.

\section{References}

Anagnos T. and Kiremidjan A.S. 1988. A review of earthquake occurrence models for seismic hazard analysis, Probabilist. Eng. Mech., 3(1), 3-11. 
Angelier J., Lyberis N., LePichon X. and Hucho X. 1982. The tectonic development of the Hellenic arc and the Sea of Crete: A synthesis, Tectonophysics, 86, 159-196.

Argus D.F., Gordon R.G., DeMets C. and Stein S. 1989. Closure of the Africa-Eurasia-North America plate motion circuit and tectonics of the Gloria fault, J. Geophys. Res., 94, 55855602.

Billingsley P. 1961. Statistical Inference for Markov Processes. The University of Chicago, Chicago Press.

Barlow R.E., Marshall A.W. and Proschan F., 1963. Properties of Probability Distributions with Monotone Hazard Rate, Ann. Math. Stat., 34, 375-389.

Barlow R.E., and Proschan F. 1975. Statistical Theory of Reliability and Life Testing, Probability Models, Holt, Rinehart and Winston, USA.

Roy D. and Gupta R.P. 1992. Classification of Discrete Lives, Microelectron. Reliab., 32, 14591473.

Bracquemond C. 2001. Modélisation Stochastique du Vieillissement en Temps Discret, Thesis, Laboratoire de Modélisation et Calcul, Grenoble.

Bracquemond C., Gaudoin O. and Xie M. 2001. Towards a New Definition of Failure Rate for Discrete Distributions, $10^{\text {th }}$ International Symposium on Applied Stochastic Models and Data Analysis, 1, 266-270.

Efron B. and Tibshirani R.J. 1993. An introduction to the bootstrap. New York: Chapman and Hall.

Jackson J. 1994. Active tectonics of the Aegean region, Annu. Rev. Earth Planet. Sci., 22, 239271.

Govers R., and Wortel M.J.R. 2005. Lithosphere tearing at STEP faults: Response to edges of subduction zones, Earth Planet. Sci. Lett., 236, 505-523.

Kemeny J.G. and Snell J.L. 1976. Finite Markov chains, Springer-Verlag, NY.

LePichon,X. and Angelier J. 1979. The Hellenic arc and trench system: A key to the neotectonic evolution of the eastern Mediterranean area, Tectonophysics, 60, 1-42.

McKenzie D. 1978. Active tectonics of the Alpine-Himalayan belt: The Aegean Sea and surrounding regions, Geophys. J. Roy. Astron. Soc., 55, 217-254.

Olivet J.L., Bonnin J., Beuzart P. and Auzende J.M. 1982. Cinématique des plaques et paléo géographie: une revue, Bull. Soc. Géol. France, 24, 875-892.

Papadimitriou E.E. 1994. Long-term earthquakes prediction in the north Pacific seismic zone based on the time and magnitude predictable model, Natural Hazards, 9, 303-321.

Papadimitriou E.E. and Karakostas V.G. 2008. Rupture model of the great AD 365 Crete earthquake in the southwestern part of the Hellenic Arc, Acta Geophysica, 56 (2), 293-312.

Papazachos B.C. and Comninakis P.E. 1971. Geophysical and tectonic features of the Aegean Arc, J. Geophys. Res. 76, 8517-8533.

Papazachos B.C. and Papazachou C. 2003. The Earthquakes of Greece, Ziti Publ., Thessaloniki, $317 \mathrm{pp}$.

Reid H.F. 1910. The mechanism of the earthquake, in: The California earthquake of April 18, 1906, report of the state earthquake Investigation Commission, Washington, D.C., Carnegie Institution, 2, 1-92.

Sadek A. and Limnios N. 2002. Asymptotic properties for maximum likelihood estimators for reliability and failure rates of Markov chains, Commun. Stat. A.-Theor., 31 (10), 1837-1861.

Scordilis E.M., Karakaisis G.F., Karakostas, B.G., Panagiotopoulos D.G., Comninakis P.E. and Papazachos B.C. 1985. Evidence for transform faulting in the Ionian Sea: The Cephalonia Island earthquake sequence, Pure Appl. Geophys., 123, 388-397.

Tsapanos T.M. and Papadopoulou A. 1999. A discrete Markov model for earthquake occurren-ces in Southern Alaska and Aleutian Islands, J. Balkan Geophys. Soc., 2 (3), 75-83.

Wortel M.J.R. and Spakman W. 1992. Structure and dynamics of subducted lithosphere in the Mediterranean region, Proc. Kon. Ned. Acad. Sci., 95, 325-347. 